\title{
Analysis of Human Lenses by Raman Microspectroscopy
}

\author{
C. Paluszkiewicz ${ }^{a, *}$, P. Chaniecki ${ }^{b}$, M. RęKas ${ }^{c}$, B. Rajchel ${ }^{a}$, N. Piergies ${ }^{a}$ \\ AND W.M. KWIATEK ${ }^{a}$ \\ ${ }^{a}$ Institute of Nuclear Physics, Polish Academy of Sciences, E. Radzikowskiego 152, 31-342 Kraków, Poland \\ ${ }^{b}$ 5th Military Hospital with Polyclinic in Kraków, Department of Ophthalmology, 30-091 Kraków, Poland \\ ${ }^{c}$ Military Medical Institute, Department of Ophthalmology, 04-141 Warszawa, Poland
}

\begin{abstract}
A cataract is an opacity (clouding) of the normally clear lens which develops as a result of aging, metabolic disorders, trauma or heredity. The number of patients with cataract is increasing exponentially. This disease requires surgical intervention, to remove the cloudy lens and to introduce the eye lens polymer. In this work we will present analyses of degraded parts of human lens. Experimental materials were obtained from the lens removed during surgical intervention. These biological samples were measured using Almega XR Confocal Raman spectrometer (Thermo Scientific) with an excitation source of $785 \mathrm{~nm}$ laser line. The Raman vibrations in the spectral region of $650-1750 \mathrm{~cm}^{-1}$ were analyzed. The difference spectra revealed an excess of tryptophan, tyrosine, phenylalanine, $\beta$-sheet conformation, and molecules or molecular groups.
\end{abstract}

DOI: 10.12693/APhysPolA.129.244

PACS: 87.64.kp, 42.55.Ye, 87.64.-t

\section{Introduction}

One of the popular eye diseases is cataract. A cataract is a clouding, observed in the crystalline lens, which decreases visual perception [1]. Possible cataract formation is associated with conformational protein changes, aggregates formation, or changes in backbone. Moreover, the significant role in cataract development are considered changes in protein side-chains such as tryptophan and tyrosine residues $[2,3]$. Each year, the number of patients with cataract is increasing exponentially. At present, the treatment for cataract disease is surgical intervention, in order to remove the cloudy lens and to introduce the eye lens polymer [3].

For better understanding the process of cataract development it is crucial to know and understand the changes in physicochemical events that characterize this eye disease formation [4]. The Raman spectroscopy seems to be a perfect tool for analyzing the lens pathological processes at a molecular level. This rapid and nondestructive spectroscopic method provides possibilities to investigate the secondary structure of lens proteins $[4,5]$.

In this paper we present the Raman spectroscopy studies of lens with cataract formation of two patients (female and male), urgently admitted to the Clinic in Warsaw in order to undergo a cataract surgery of the eye. The material consisted of the lenses differed in their degree of fragmentation as a result of the use of ultrasound.

\section{Materials and methods}

As it was mentioned in the previous paragraph, the cataract lens samples were obtained from two patients in

\footnotetext{
* corresponding author; e-mail: czeslawa.paluszkiewicz@ifj.edu.pl
}

different age (75 years old female and 83 years old male). The lenses were extracted with the phacoemulsification method and put in the cassettes with peristatic membrane. After surgery the cassettes with removed lenses were sent to IFJ PAN Kraków for further investigations. Before the Raman measurements, the specimen from the surgical medium was extracted and centrifuged. The samples, obtained in that way, were dried in open air atmosphere. The Raman spectra were recorded by Almega XR Raman spectrometer (Thermo Scientific) equipped with a confocal microscope in combination with the 100 magnification objective and CCD detector. As an excitation source the $785 \mathrm{~nm}$ laser line with exposure time of $20 \mathrm{~s}$, was used. The Raman vibrations in the spectral region of $650-1750 \mathrm{~cm}^{-1}$ with resolution $4 \mathrm{~cm}^{-1}$, were analyzed.

\section{Results and discussion}

Because the biological membranes contain well-defined composition, which is changing with age and cataract formation, many scientists indicate that the lipid composition alterations are crucial for recognition human lens clarity [6]. However, the changes observed in the fingerprint region, where information about secondary structure of lens proteins and amino acid residues are provided, also show some differences with cataracts aggravation [1]. Thus, we decided to perform carefully investigations of the Raman spectra of those two lenses with cataract in the spectral region of $650-1750 \mathrm{~cm}^{-1}$.

Figure 1 presents the Raman spectrum obtained from degraded human lens of 83 years old man and Fig. 2 shows the Raman spectra obtained from degraded human lens of 75 years old female (measured in two points: $\mathrm{A}$ and $\mathrm{B}$ ). In these spectra one can see bands due to the amide I $\left(\approx 1670 \mathrm{~cm}^{-1}\right)[7,8]$, tryptophan/tyrosine/phenylalanine $\left(\approx 1614 \mathrm{~cm}^{-1}\right)[9-$ 12], amide III $\left(\approx 1246 \mathrm{~cm}^{-1}\right)$ [7, 8], phenylalanine 


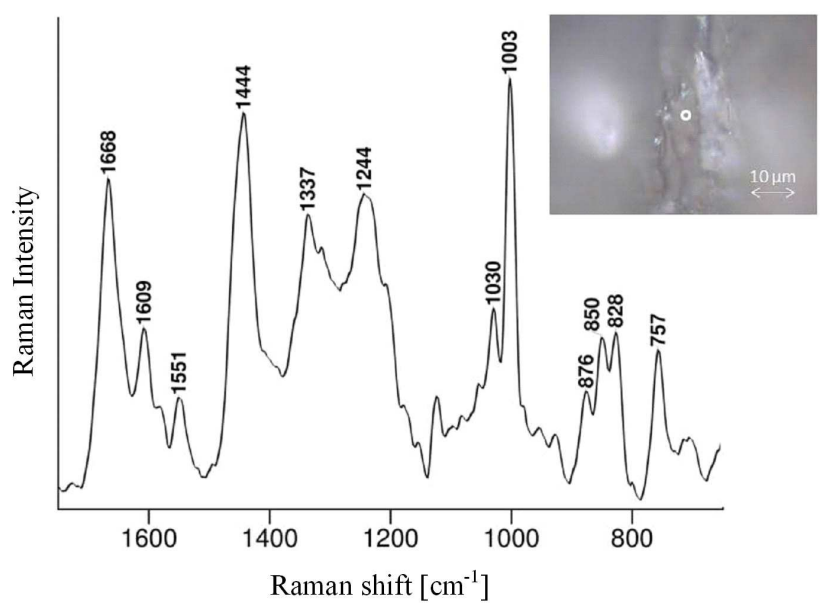

Fig. 1. Raman spectrum obtained from degraded human lens (man) together with the image of the investigated area.

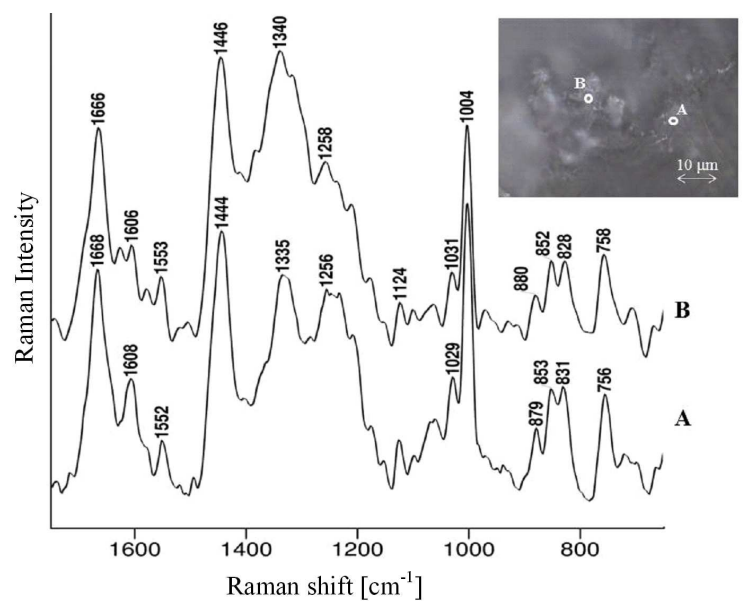

Fig. 2. Raman spectra obtained from degraded human lens (point A and B) (female) together with the image of the investigated areas.

$\left(\approx 1033 \mathrm{~cm}^{-1}\right.$ and $\left.\approx 1000 \mathrm{~cm}^{-1}\right)[9,12]$, tryptophan $\left(\approx 875 \mathrm{~cm}^{-1}\right.$ and $\left.\approx 757 \mathrm{~cm}^{-1}\right)$ [11], and tyrosine $(\approx$ $852 \mathrm{~cm}^{-1}, \approx 830 \mathrm{~cm}^{-1}$, and $\left.\approx 643 \mathrm{~cm}^{-1}\right)$ [10] vibrations (see Figs. 1 and 2 for precise wave numbers). Briefly, for all Raman spectra (Figs. 1,2) the amide I and amide III bands appear at $\approx 1670 \mathrm{~cm}^{-1}$ and $\approx 1246 \mathrm{~cm}^{-1}$. Position of the aforementioned vibrational bands is significant for determining the conformational protein changes [13]. The observed amide I and amide III spectral pattern suggests that the proteins of investigated human lens are mainly in the $\beta$ sheet conformation [14].

On the other hand, the tryptophan and tyrosine residue bands are very sensitive for any lens opacity changes [1]. For example, the weakly enhanced Raman band at $875 \mathrm{~cm}^{-1}$ due to the tryptophan vibrations proves that the tryptophan conformation is "exposed", namely the $-\mathrm{OH}$ bands from the tryptophan benzene ring combined with $\mathrm{H}_{2} \mathrm{O}$. This is compatible with the previous published results $[1,13]$ which have indicated that during the lens opacification the tryptophan residue changes from "buried" to "exposed" conformation. Moreover, the tyrosine doublet $\left(\approx 852 \mathrm{~cm}^{-1}\right.$ and $\left.\approx 830 \mathrm{~cm}^{-1}\right)$ which determines the nature of the hydrogen bonding observed in the phenolic hydroxyl in the tyrosine residue [10], is also sensitive for alteration provided by cataracts formation [1]. In the Raman spectra of male lens (Fig. 1) and female lens from position A (Fig. 2) the ratio of $I_{\approx 850 \mathrm{~cm}^{-1}} / I_{\approx 828 \mathrm{~cm}^{-1}}$ (see Figs. 1 and 2 for precise wave numbers) is equal to 0.97 and 0.98 , respectively. This spectral pattern shows that tyrosine residues, which usually create hydrogen bond with $\mathrm{H}_{2} \mathrm{O}$, are strongly hydrogen bonded to acceptors for these two samples. This "buried" conformation of tyrosine is observed during lens opacification. On the other hand, in the Raman spectrum of female lens at position B (Fig. 2) the opposite phenomenon, is noticed. Here, the ratio $I_{852 \mathrm{~cm}^{-1}} / I_{828 \mathrm{~cm}^{-1}}$ is equal to 1.11, which suggests that the tyrosine residue is strong bonded to $\mathrm{H}_{2} \mathrm{O}$ via hydrogen bond $[1,10]$. Such spectral changes observed for the female lens at two different position (A and B, see Fig. 2) may indicate that the progress of degradation does not proceed uniformly over the entire volume of the lens.

\section{Conclusions}

Observed spectral patterns of the investigated samples indicate that the Raman spectroscopy can be applied to study lenses degradation. Moreover, the changes in spectral intensity may be related to the alteration in the tryptophan and tyrosine conformation, which can be different in various volumes of lens sample. However, the above mentioned alteration may be also associated with the fragmentation of lenses prompted by the use of ultrasound during phacoemulsification surgery. In order to confirm the above conclusions several hundreds of clinical causes have to be studied.

\section{Acknowledgments}

The project has been supported by National Science Centre Poland under decision no. DEC-2012/05/B/ST4/ 01150 .

\section{References}

[1] Z. Zhuang, M. Zhu, Y. Huang, J. Liu, Z. Guo, K. Xiong, N. Li, S. Chen, X. Qiu, Appl. Phys. Lett. 101, 173701 (2012).

[2] Y. Ozaki, A. Mizuno, M. Yoshiura, T. Iwamoto, K. Iriyama, Biochemistry 22, 6254 (1983).

[3] M.H. Smeets, G.F.J.M. Vrensen, K. Otto, G.J. Puppels, J. Greve, Biochim. Biophys. Acta 1164, 236 (1993).

[4] K. Itoh, Y. Ozaki, A. Mizuno, K. Iriyama, Biochemistry 22, 1773 (1983). 
[5] N.T. Yu, E.J. East, J. Biol. Chem. 250, 2196 (1975).

[6] D. Borchman, D. Tang, M.C. Yappert, Biospectroscopy 5, 151 (1999).

[7] K. Furic, V. Mohacek-Grosev, M. Hadzija, J. Mol. Struct. 744-747, 169 (2005).

[8] J. Kong, S. Yu, Acta Biochem. Biophys. Sin. 39, 549 (2007).

[9] S.-Y. Lin, K.-H. Chen, Ch.-Ch. Lin, W.-T. Cheng, M.J. Li, Spectrochim. Acta A 77, 703 (2010).

[10] M.N. Siamwiza, R.C. Lord, M.C. Chen, T. Takamatsu, I. Harada, H. Matsuura, T. Shimanouchi, Biochemistry 14, 4870 (1975).
[11] A.E. Aliaga, I. Osorio-Roman, P. Leyton, C. Garrido, J. Carcamo, C. Caniulef, F. Celis, G. Diaz F., E. Clavijo, J.S. Gomez-Jeria, M.M. Campos-Vallette, J. Raman Spectrosc. 40, 164 (2009).

[12] B. Ravikumar, R.K. Rajaram, V. Ramakrishnan, J. Raman Spectrosc. 37, 597 (2006).

[13] P.R. Carey, V.R. Salares, Adv. Infrared Raman Spectrosc. 7, 1 (1980).

[14] D.M. Thomas, K.L. Schepler, Invest. Ophth. Vis. Sci. 19, 904 (1980). 